\title{
VIBRATONAL MECHANICS AND STOCHASTIC QUASI-RESONANCES
}

\author{
Eugen Kremer \\ Laboratory of Vibrational Mechanics \\ IPME RAS \\ Russia \\ keb@ipme.ru
}

Article history:

Received 01.09.2021, Accepted 27.09.2021

\begin{abstract}
The concept of vibrational mechanics was pioneered in the works by Professor I.I. Blekhman and developed by his numerous disciples and coleagues. It is a powerful tool for the study of such systems with fast excitations, in which slow motion is of primary interest. One important application of this approach is the stochastic resonance, the phenomenon of resonance-like response of slow variables to intensity of stochastic excitation. This phenomenon is considered within the framework of vibrational mechanics as forced lowfrequency oscillations near the natural frequency, which evolves under the influence of changing high-frequency stochastic excitation. We propose a generalization of this approach to the case when the evolution of low-frequency properties of the system leads not to the equality of the natural frequency and the frequency of the external slow force, but to the loss of stability in a certain interval of the stochastic excitation intensity. Since in this case, as for stochastic resonance, the external manifestation of the process is the resonance-like response of the system, the considered effect can be called stochastic quasi-resonance, As an example, we consider a rotor with anisotropy of bending stiffness under the action of stochastic angular velocity oscillations.
\end{abstract}

\section{Key words}

Vibrational mechanics, Rotor dynamics, Stochastic resonance, Stochastic quasi-resonance, Rotor anisotropy, Separation of motions

\section{Introduction}

The scientific heritage of Professor I.I. Blekhman is outstanding not only for the richness, variety and scientific significance of his results, but also for the fruitful ideas that he generously shared with his disciples and colleagues, giving origin to new promising scientific directions.

One of his most important fundamental ideas, the concept of vibrational mechanics, became the basis not simply of a new direction, but in fact of a new branch of mechanics [Blekhman, 2000; Blekhman, 2004; Blekhman, 2012]. The essence of vibrational mechanics consists in the replacement of the initial system with high-frequency actions by some equivalent slow system, in which the influence of the discarded fast motions on the averaged motion is considered by introducing additional so called vibrational forces. This approach makes especially efficient and transparent the investigation of such systems with high-frequency influences, in which slow motions are of primary interest.

The vibrational mechanics approach has in turn influenced the development of the theory of stochastic resonance, a phenomenon of the resonance-like response of slow variables to the intensity of highfrequency stochastic excitation. This phenomenon was first described in [Benzi et al., 1981] and at first was considered as purely probabilistic, but gradually the connection between stochastic and vibrational resonance [Landa and McClintock, 2000] became clear.

After the pioneering work [Blekhman and Landa, 2004] a new paradigm in the interpretation of this phenomenon within vibrational mechanics began to develop [Blekhman and Sorokin, 2019; Blekhman and Kremer, 2019; Kremer, 2018; Sorokin, 2020; E.Kremer, 2021]. It is based on the fact that there is a low-frequency excitation in the system along with a high-frequency excitation. 
Since high-frequency excitations modify the low natural frequency of the system, a low-frequency resonance occurs at a certain level of their intensity.

In this paper, this concept is generalized to the case when modification of low-frequency properties of the system under the action of highfrequency excitation leads not to resonance relations but to the loss of stability in the averaged system, which also causes a resonance-like response of the system. We call this effect stochastic quasiresonance and consider it here on the example of a double-bending rotor with a single disk and highfrequency stochastic angular velocity oscillations.

\section{Rotor with double bending stiffness under stochastic excitation}

We consider the rotor shown in Fig. 1 with one disk of mass $\mathrm{M}$ mounted symmetrically and horizontally ( $\mathrm{g}$ is acceleration of gravity) on two rigid bearing supports and having different bending stiffnesses $c_{1}$ and $c_{2}$ in the two principal directions $\eta_{1}, \varsigma_{1}$ of the rotating coordinate system.

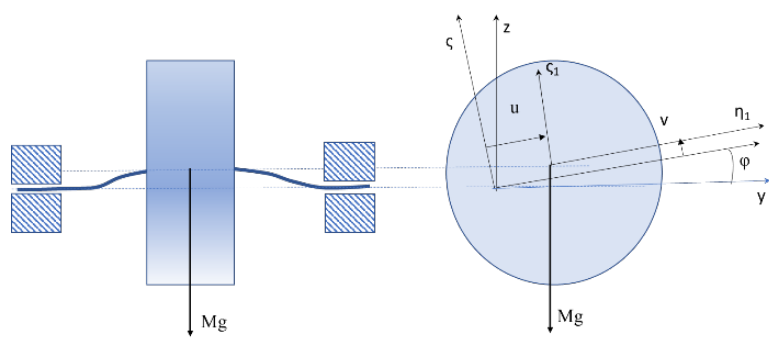

Figure 1. Rotor with double bending stiffness

In the absence of stochastic excitation this classical system is described, for example, in [Kellenberger, 1958]. Here, we will consider this rotor under stochastic oscillations of its angular velocity.

\subsection{Stochastic kinematic excitation of the rotor}

The position angle $\varphi$ of the rotating coordinate system $\eta, \varsigma$ with respect to the stationary coordinate system $y, z$ varies according to the equation

$$
\dot{\varphi}=\omega_{0}(1+\xi),
$$

where $\omega_{0}$ is the mean angular velocity of the rotor, $\xi$ is the relative dimensionless stochastic component of the angular velocity depending on the dimensionless fast time $\theta=\omega_{0} t / \varepsilon$, which differs from the slow time $\tau=\omega_{0} t$ by a factor $1 / \varepsilon$, where $\varepsilon$ is some small parametre.This means that the typical frequency in the stochastic excitation spectrum $\omega_{0} / \varepsilon$ is large compared to the rotor mean angular velocity $\omega_{0}$. It is assumed that the stochastic process $\xi$ has a canonical expansion [E.Kremer, 2021]

$$
\xi=\int_{0}^{\infty}\left(\xi_{s}(\mathrm{k}) \sin \mathrm{k} \theta+\xi_{c}(\mathrm{k}) \cos \mathrm{k} \theta\right) d \mathrm{k}
$$

where $\xi_{s}(\mathrm{k})$ and $\xi_{c}(\mathrm{k})$ are mutually independent and stochastically orthogonal random functions, namely, they have the following properties:

$$
\begin{array}{r}
E\left(\xi_{s}(\mathrm{k})\right)=E\left(\xi_{c}(\mathrm{k})\right)=E\left(\xi_{s}(\mathrm{k}) \xi_{c}(\mathrm{k})\right)=0, \\
E\left(\xi_{s}\left(\mathrm{k}_{\mathrm{i}}\right) \xi_{s}\left(\mathrm{k}_{\mathrm{j}}\right)\right)=E\left(\xi_{c}\left(\mathrm{k}_{\mathrm{i}}\right) \xi_{c}\left(\mathrm{k}_{\mathrm{j}}\right)\right)=S\left(k_{i}\right) \delta\left(k_{i}-k_{j}\right)
\end{array}
$$

Here the symbol E denotes the mathematical expectation, $\delta(k)$ is the $\delta$-function and $\mathrm{S}(\mathrm{k})$ is the spectral density of the process, which is a specified deterministic function. It is also assumed that the spectral density decreases at $k \rightarrow 0$ fast enough to ensure convergence of the integral

$$
\kappa=\int_{0}^{\infty} S(\mathrm{k}) \mathrm{k}^{-2} d \mathrm{k}
$$

As it is shown in the paper [Kremer, 2018], the value $\kappa$ is determinative for formulation of the equation of slow motions in vibrational mechanics with stochastic excitations. For further consideration, it is convenient to introduce the designation $\xi_{1}=\xi$, and also to denote the corresponding quantity $\kappa$ as $\kappa_{1}$. This is related to the fact that in formulating the equations we also need the quantity $\xi^{2}$ related to the centripetal acceleration. This quantity can be represented as $\xi^{2}=\left\langle\xi^{2}\right\rangle+\xi_{2}$, where is denoted $\xi_{2}=\xi^{2}-\left\langle\xi^{2}\right\rangle$. The stochastic process $\xi_{2}$ has the same properties Eq.(3) as the process $\xi_{1}$ but has a different spectral density, for which the value $\kappa$ calculated by Eq.(4) is denoted by $\kappa_{2}$.

Now, we proceed directly to the equations of motion for the rotor

\subsection{Equations of motion for the rotor}

The position of the centre of the disk $\mathrm{u}, \mathrm{v}$ in the moving coordinate system $\eta, \varsigma$ is described by the equations (without damping)

$$
\begin{aligned}
& M\left(\ddot{u}-2 \omega \dot{v}-\omega^{2} \mathrm{u}\right)+\mathrm{c}_{1} \mathrm{u}=-\mathrm{Mg} \sin \varphi \\
& M\left(\ddot{v}+2 \omega \dot{u}-\omega^{2} \mathrm{v}\right)+\mathrm{c}_{2} \mathrm{v}=-\mathrm{Mg} \cos \varphi
\end{aligned}
$$

Eqs. (5) and (1) represent a fifth-order system, which can be reduced to the form

$$
\dot{x}_{i}=F_{i}+\sum_{j=1}^{m} B_{i j} \xi_{j},
$$


proposed in [E.Kremer, 2021] as a standard one for deriving the equations of slow motions for a system of order $\mathrm{n}$ with $\mathrm{m}$ independent stochastic processes $\xi_{j}$ in the excitation. Here $F_{i}$ and $B_{i j}$ are functions of $x_{s}(s=1 . . n)$ which do not depend explicitly on high-frequency excitations. In the problem under consideration, $\mathrm{n}=5, \mathrm{~m}=2$, and the variables $x_{i}$ can be introduced, for example, as follows:

$x_{1}=\frac{\omega_{0}^{2} u^{\prime}}{g}, x_{2}=\frac{\omega_{0}^{2} v^{\prime}}{g}, x_{3}=\frac{\omega_{0}^{2} u}{g}, x_{4}=\frac{\omega_{0}^{2} v}{g}, x_{5}=\varphi$

Here the dash denotes differentiation by dimensionless time $\tau=\omega_{0} t$. The introduction of variables by Eq. (7) results in dimensionless parameters

$$
\lambda_{1}=\sqrt{\frac{c_{1}}{M \omega_{0}^{2}}}, \quad \lambda_{2}=\sqrt{\frac{c_{2}}{M \omega_{0}^{2}}}
$$

The functions $F_{i}$ and $B_{i j}$ are

$$
\begin{gathered}
F_{1}=2 x_{2}+\left(1-\lambda_{1}^{2}+\left\langle\xi^{2}\right\rangle\right) x_{3}-\sin x_{5}, \\
F_{2}=-2 x_{1}+\left(1-\lambda_{2}^{2}+\left\langle\xi^{2}\right\rangle\right) x_{3}-\cos x_{5}, \\
F_{3}=x_{1}, F_{4}=x_{2}, F_{5}=1 ; B_{11}=2 x_{2}+2 x_{3}, \\
B_{12}=x_{3}, B_{21}=-2 x_{1}+2 x_{4}, B_{22}=x_{4} \\
B_{31}=B_{41}=B_{32}=B_{42}=B_{52}=0, B_{51}=1
\end{gathered}
$$

\subsection{Equations for slow motion of the rotor}

In the paper [E.Kremer, 2021] within the framework of vibrational mechanics, the equations of slow motions for the general case of stochastic excitation of the form (6) were derived. We concretize this result for the considered case of a fifth-order system with two stochastic processes $\xi_{1}$ and $\xi_{1}$. Here, the slow motion equations take the form

$$
X_{i}^{\prime}=\overline{F_{i}}+\varepsilon^{2}\left(\kappa_{1} V_{i 1}+\kappa_{2} V_{i 2}\right), \mathrm{i}=1 . .5
$$

with $X_{i}=\left\langle x_{i}\right\rangle, \overline{F_{i}}=F_{i}\left(X_{1}, . . X_{5}\right)$, and the functions $V_{i j}(\mathrm{j}=1,2)$ are calculated by the formula which for linear equations is of the form

$$
V_{i j}=\sum_{k=1}^{5} \frac{\partial \bar{B}_{i j}}{\partial X_{k}}\left(\bar{B}_{k j}-\sum_{s=1}^{5} \frac{\partial \bar{F}_{k}}{\partial X_{s}} \bar{B}_{s j}\right)
$$

where denoted $\overline{B_{i}}=B_{i}\left(X_{1}, . . X_{5}\right)$.

Considering formulas (9) and introducing new slow variables $\mathrm{X}, \mathrm{Y}$ and parameters $\gamma, \sigma, v$ and $\beta$ by formulas

$$
\begin{array}{r}
X=X_{3}, Y=X_{4}, \\
\gamma=4 \varepsilon^{2} \kappa_{1}, \sigma=\left\langle\xi^{2}\right\rangle-4 \varepsilon^{2} \kappa_{2}, \\
v=\lambda_{1}^{2}-1, \beta=\frac{\lambda_{2}^{2}-1}{\lambda_{1}^{2}-1}
\end{array}
$$

we obtain the equations of slow motion in the following compact form

$$
\begin{array}{r}
X^{\prime \prime}-(2+\gamma) Y^{\prime}+\gamma X^{\prime}+(\nu-\gamma-\sigma) X-\gamma Y=-\sin \tau \\
Y^{\prime \prime}+\gamma Y^{\prime}+(2+\gamma) X^{\prime}+\gamma X+(\nu \beta-\gamma-\sigma) Y=-\cos \tau
\end{array}
$$

\section{Stability analysis and stochastic quasi-resonance}

Slow motions in the rotating coordinate system according to equations (13) consist of forced oscillations under the action of gravity with a dimensionless frequency 1 and of free oscillations determined by the homogeneous part of the system. Further, we investigate the conditions under which free vibrations are damped, i.e. we analyze the stability of the system as a function of the intensity of the stochastic excitation

\subsection{Domains of stability}

We will consider the case where the system is stable in the absence of stochastic excitation. For this, the angular velocity must lie outside the interval between the partial frequencies of the rotor, i.e. the difference of $\lambda_{1}$ and $\lambda_{2}$ from 1 must have the same signs. According to Eq.(12), this is ensured by the condition $\beta \geq 0$. The pre-critical rotational speed corresponds to $v \geq 0$, and the supercritical one to $v \leq 0$. We will analyze the stability in the plane of parameters $p=\gamma /|v|$ and $q=\sigma /|v|$. Note that according to Eq.(12) only the case $p \geq 0$ has a physical sense.

The characteristic equation of the system is

$$
a_{0} s^{4}+a_{1} s^{3}+a_{2} s^{2}+a_{3} s+a_{4}=0
$$

with the coefficients $a_{0}=1, a_{1}=2 \gamma, a_{2}=2 \gamma^{2}+2 \gamma+$ $4+v(1+\beta)-2 \sigma, a_{3}=\gamma\left(v(1+\beta)+2(2-\sigma)\right.$ and $a_{4}=$ $(\gamma+\sigma)^{2}-(\gamma+\sigma)(1+\beta) v+v^{2} \beta+\gamma^{2}$.

It is easy to show that for small $v$ all the inequalities of the Routh- Hurwitz criterion are automatically fulfilled, with the exception of the condition $a_{4} \geq 0$, which defines the stability boundaries in the region of parameters $p$ and $q$.

These boundaries are depicted for the cases $v \geq 0$ and $v \leq 0$ in Fig. 2 and Fig. 3 (regions of instability are hatched) and for various values of $\beta$.

\subsection{Stochastic quasi-resonance}

We will consider the evolution of the system with increasing stochastic excitation using the diagram in Fig. 2 (pre-critical rotation speed). In the absence of excitation $(\mathrm{p}=\mathrm{q}=0)$, the system is stable. Increasing excitation means increasing $\mathrm{p}$ and $\mathrm{q}$ along some curve in the plane of these parameters. Whatever this curve is, the system inevitably enters the region of instability, and with further increase in excitation inevitably leaves it. Thus, 


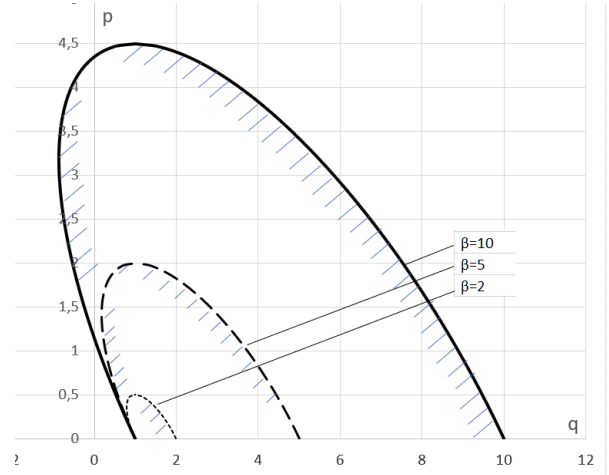

Figure 2. Areas of rotor instability in the stochastic excitation parameter plane $(p, q)$ for a supracritical rotor speed $(v \geq 0)$

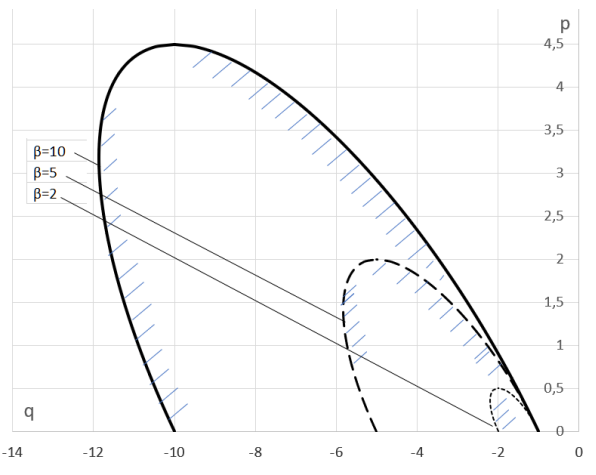

Figure 3. Areas of rotor instability in the stochastic excitation parameter plane $(p, q)$ for a supracritical rotor speed $(v \leq 0)$

there is a resonance-like response of slow motions to the intensity of the stochastic excitations. We call it stochastic quasi-resonance, emphasising that the nature of this phenomenon is different from the usual resonance. For the supercritical situation (Fig.3), a stochastic quasi-resonance is in principle also possible, since negative values of $\mathrm{q}$ are available, but in this case it is realized only under certain special scenarios of increasing stochastic excitation and, in contrast to the pre-critical case, it is not inevitable.

\section{Conclusion}

On the example of an anisotropic rotor with high-frequency stochastic angular velocity oscillations, the possibility of a peculiar phenomenon resembling stochastic resonance is shown. This phenomenon can be called a stochastic quasiresonance, because in contrast to the stochastic resonance its origin is not resonance-frequency relations, but the passage of instability region for slow motion . Note that stochastic quasi-resonance, unlike stochastic resonance, does not require lowfrequency excitation

In the future it is of interest to consider the phe- nomenon of quasi-resonance in a more general formulation and to identify its further applications

\section{Acknowledgements}

The idea for this study as for many other papers by the author could hardly have originated without the enormous influence of the communication and joint work he had with Professor I. I. Blekhman. This article is dedicated to his blessed memory.

\section{References}

Benzi, R., Sutera, A., and Vulpiani, A. (1981). The mechanism of stochastic resonance. Journal of Physics A: Mathematical and General, 14(11), pp. L453-L457.

Blekhman, I. (2000). Vibrational Mechanics: Nonlinear Dynamic Effects, General Approach, Applications. World Scientific, Singapore.

Blekhman, I. (2004). Selected Topics in Vibrational Mechanics -. World Scientific, Singapur.

Blekhman, I. (2012). Oscillatory strobodynamics a new area in nonlinear oscillations theory, nonlinear dynamics and cybernetical physics. Cybernetics and Physics, 1 (1), pp. 5-10.

Blekhman, I. and Kremer, E. (2019). Stochastic resonance as the averaged response to random broadband excitation and its possible applications. Proceedings of the Institution of Mechanical Engineers, Part C: Journal of Mechanical Engineering Science, 233 (23-24), pp. 7476-7488.

Blekhman, I. and Landa, P. (2004). Conjugate resonances and bifurcations in nonlinear systems under biharmonical excitation. International Journal of Non-Linear Mechanics, 39 (3), pp. 421-426.

Blekhman, I. and Sorokin, V. (2019). On the stochastic resonance phenomenon in parametrically excited systems. European Journal of Applied Mathematics, 30 (5), pp. 986-1003.

E.Kremer (2021). The effect of high-frequency stochastic actions on the low-frequency behaviour of dynamic systems. Phil. Trans. R. Soc. A., 379 (2192), pp. 2020-0242.

Kellenberger, W. (1958). Biegeschwingungen einer unrunden, rotierenden welle in horizontaler lage. Ingenieur-Archiv, 26 (4), pp. 1432-0681.

Kremer, E. (2018). Low-frequency dynamics of systems with modulated high-frequency stochastic excitation. Journal of Sound and Vibration, 437, pp. 422-436.

Landa, P. S. and McClintock, P. V. E. (2000). Vibrational resonance. Journal of Physics A: Mathematical and General, 33 (45), pp. L433-L438.

Sorokin, V. (2020). Vibrations of a nonlinear stochastic system with a varying mass under near resonant excitation. Journal of Vibration and Control, 26 (17-18), pp. 1435-1444. 